\title{
Médiévales
}

Langues, Textes, Histoire

49 | automne 2005

La paroisse, genèse d'une forme territoriale

\section{Paroisse, paroissiens et territoire. Remarques sur parochia dans les textes latins du Moyen Âge}

Parish, parishioners and territories. Remarks on the word " parochia » in medieval Latin sources.

\section{Michel Lauwers}

\section{OpenEdition}

Journals

Édition électronique

URL : https://journals.openedition.org/medievales/1260

DOI : 10.4000/medievales. 1260

ISSN : $1777-5892$

Éditeur

Presses universitaires de Vincennes

Édition imprimée

Date de publication : 1 décembre 2005

Pagination : 11-32

ISBN : 2-84292-177-1

ISSN : 0751-2708

\section{Référence électronique}

Michel Lauwers, « Paroisse, paroissiens et territoire. Remarques sur parochia dans les textes latins du Moyen Âge », Médiévales [En ligne], 49 | automne 2005, mis en ligne le 26 mars 2008, consulté le 22 avril 2022. URL : http://journals.openedition.org/medievales/1260; DOI : https://doi.org/10.4000/ medievales. 1260

Ce document a été généré automatiquement le 22 avril 2022

Tous droits réservés 


\title{
Paroisse, paroissiens et territoire. Remarques sur parochia dans les textes latins du Moyen Âge
}

Parish, parishioners and territories. Remarks on the word "parochia » in medieval Latin sources.

\author{
Michel Lauwers
}

Pendant longtemps, les historiens ont envisagé la paroisse, de manière presque naturelle, comme la circonscription ecclésiastique de base au sein d'une hiérarchie administrative et territoriale qui se serait mise en place au moment de la christianisation de l'Empire romain. Ils ont donc entrepris d'en faire l'histoire depuis les origines, au IV ${ }^{\mathrm{e}}$ siècle, jusqu'à la fin du Moyen Âge ${ }^{1}$. Le modèle dominant était alors celui de la "grande paroisse » ou "paroisse primitive» du haut Moyen Âge dont se seraient progressivement détachées des paroisses nouvelles ou secondaires, de taille plus réduite. Ainsi les médiévistes évoquaient-ils des "paroisses» mérovingiennes, carolingiennes, $\mathrm{du} \mathrm{XI}^{\mathrm{e}}$ ou du XIII ${ }^{\mathrm{e}}$ siècle, confortés par la présence de parochiae dans tous les textes de ces époques.

2 À partir des années 1980, plusieurs travaux ont toutefois attiré l'attention sur les phénomènes de rupture et de réorganisation qui ont marqué l'histoire médiévale du peuplement et de l'habitat, en soulignant notamment la dispersion et la fluidité des sites ruraux du haut Moyen Âge, sinon la déliquescence de la plupart des structures territoriales propres au monde antique, puis un lent mouvement de fixation des populations, en milieu rural, autour des églises et des aires funéraires. Or ce processus de fixation que Robert Fossier a désigné comme étant celui de la «naissance du village » paraît s'être tout d'abord manifesté par une "naissance de la paroisse », cette dernière étant en quelque sorte la face visible du village. La paroisse n'était donc plus, dans cette perspective, une réalité immuable, intrinsèque à l'organisation de la société chrétienne ; elle renvoyait à un phénomène complexe, caractérisé par trois éléments : le regroupement et l'ancrage des hommes à proximité des lieux de culte, la formation 
d'entités territoriales correspondant à ces regroupements, et enfin le maillage des territoires paroissiaux ainsi constitués dans un monde désormais plein.

Une sorte de consensus semble aujourd'hui régner parmi les historiens médiévistes pour réserver la notion de "paroisse " à l'entité territoriale correspondant à une communauté de fidèles ${ }^{2}$. Cet usage, qui ne semble toutefois pas s'être imposé chez tous les archéologues ni auprès des spécialistes de l'Antiquité chrétienne ${ }^{3}$, ne doit pas masquer l'existence de réelles divergences, parmi les chercheurs, dans l'appréciation de la chronologie et des modalités du processus de territorialisation. Un certain nombre d'hypothèses sont avancées à ce propos dans ce numéro de Médiévales. Ma contribution donnera le point de vue de l'historien des textes confronté aux définitions médiévales et, de manière plus large, au vocabulaire de la paroisse : dans quelle mesure les usages du mot parochia renvoyaient-ils à une dimension territoriale? Ces usages indiquent-ils des infléchissements au cours du Moyen Âge?

Un petit exemplum suffira à illustrer toute l'importance des questions de vocabulaire, ainsi que la prudence dont il convient de faire preuve dans l'interprétation des textes. Les historiens qui soutiennent le caractère précoce de la mise en place des territoires paroissiaux invoquent notamment un canon du concile réuni à Tours en 461 . Selon une traduction récemment proposée de ce canon, «il est bon de surveiller qu'un évêque ne tente de faire intervenir son autorité dans le territoire du ressort de son frère, de façon à pouvoir s'adjoindre des églises paroissiales extérieures à son diocèse, alors que les limites [des diocèses] ont été décidées par les Pères ${ }^{4}$. On en déduit que diocèses et paroisses constituaient, dans la Gaule du ve siècle, des territoires délimités, bornés et stables. Cependant, si l'on retourne au texte latin du canon de Tours, on s'aperçoit, d'une part, qu'il n'y est aucunement question de "paroisse " ni même d'«église paroissiale » et que, d'autre part, la traduction que je viens de citer donne à ce canon un sens spatial qui force peut-être le texte. En fait, le concile de Tours recommande de surveiller les éventuelles interventions $d u$ "pouvoir » d'un évêque au détriment des prérogatives de l'un de ses "frères ", ainsi que l'invasion par un évêque de "diocèses » qui ne sont pas les siens, car une telle attitude, est-il précisé, reviendrait à «transgresser les bornes mises par les pères " ${ }^{5}$. Cette dernière expression (transgredere terminos a patribus constitutos), qui rappelle le vocabulaire antique de l'arpentage et pourrait renvoyer à une procédure de bornage, est surtout une réminiscence de l'Écriture Sainte ${ }^{6}$, fréquemment attestée dans les textes du haut Moyen Âge, le plus souvent sans connotation spatiale- l'expression est généralement employée de manière polémique, parfois pour condamner les « nouveautés » sur un plan doctrinal 7.

Qu'en est-il dans les textes où figure bien le mot parochia ? Il n'est pas possible, dans le cadre de cette brève contribution, de mener une véritable étude sémantique relative à la notion de paroisse ; je donnerai seulement quelques jalons en vue d'une telle étude, bien conscient que le parcours à travers les textes canoniques et les chartes que je propose dans les pages qui suivent est semé d'embûches, qui tiennent tout à la fois à la variété du lexique selon les régions et les époques (on a remarqué, par exemple, que le terme parochia est fréquent dans les conciles gaulois, mais exceptionnel dans les conciles africains, qui lui préfèrent plebs), au problème de l'articulation des mots entre eux (un mot n'a de sens que dans un contexte, par rapport à d'autres mots) et à la question de la typologie documentaire (comme en témoigne le fréquent décalage entre le vocabulaire des actes dits de la pratique et celui, plus conservateur, des textes normatifs) ${ }^{8}$. 
Lorsque l'on examine les centaines d'occurrences médiévales du mot parochia (attesté dans le vocabulaire chrétien à partir $d u I^{e}$ siècle), un premier constat s'impose : fortement polysémique, ce terme renvoie à des réalités très différentes. Les dictionnaires de latin médiéval proposent les sens de "province ecclésiastique ", " diocèse ", " paroisse ", etc., soit à peu près toutes les formes possibles de territoires ecclésiaux, en même temps que communauté d'habitants, membres d'une paroisse, et ensemble des droits paroissiaux ${ }^{9}$.

7 Dans les contextes où ce sont plusieurs termes, hiérarchisés, qui renvoient à des lieux ou à des circonscriptions ecclésiastiques, parochia désigne en général l'échelon élémentaire, et fréquemment l'église rurale par rapport à l'église de la cité : le couple parochia vs. ciuitas est attesté dans plusieurs documents du haut Moyen Âge ${ }^{10}$, et la parochia se trouve parfois explicitement qualifiée de rustica ou rusticana ${ }^{11}$. Pour autant, parochia semble toujours désigner un lieu de culte contrôlé par la hiérarchie ecclésiastique, bien distinct des églises privées ou patrimoniales qui sont plutôt nommées « chapelles » ou « oratoires ${ }^{12}$.

Ce type d'usage de parochia est cependant loin de représenter toutes les occurrences du terme. Le mot était aussi fréquemment employé pour qualifier la communauté des fidèles rassemblée autour de son prêtre ou de son évêque, et, dans ce dernier cas, il se rapporte davantage à notre diocèse. L'utilisation de parochia pour désigner un diocèse est encore courante au $\mathrm{xI}^{\mathrm{e}}$ siècle, par exemple chez un Sigebert de Gembloux. Inversement, dans le haut Moyen Âge, diocesis, peu courant, avait désigné une subdivision de la parochia. Ces usages lexicaux faisaient dire à Victor Saxer qu'à haute époque, «la parochia, c'est le diocèse » et que «la diocesis, c'est la paroisse », l'emploi des termes étant "exactement contraire à celui d'aujourd'hui» ${ }^{13}$. Ce paradoxe n'explique toutefois pas grand-chose, surtout si l'on considère qu'il y a toujours eu des paroisses et que seules ont changé les conventions lexicales par lesquelles celles-ci étaient désignées. Il est préférable de ne pas poser a priori l'existence de territoires paroissiaux (que ceux-ci soient qualifiés par un terme ou un autre). On chercherait en vain, du reste, des attestations claires de tels territoires dans les textes du haut Moyen Âge. L'image qui en ressort est plutôt celle d'un système hiérarchisé de relations personnelles, mis au point et contrôlé par l'institution ecclésiale, supposant la soumission à l'évêque de prêtres et de groupes de fidèles.

«Plebs » et circuits paroissiaux

Dans l'Antiquité tardive et le haut Moyen Âge, la paroisse est essentiellement une assemblée (conuentus) ou un peuple de fidèles (plebs ou populus). Les évêques réunis en 506 au concile d'Agde évoquent, en effet, l'«assemblée légitime et ordinaire » qui constitue les paroisses ${ }^{14}$. Quelques années plus tôt, le pape Gélase $\mathrm{I}^{\mathrm{er}}$, appelant les évêques d'Italie à ne pas bouleverser l'«état des paroisses", avait défini celles-ci comme les assemblées d'«un peuple dévot en vue de la régénération [c'est-à-dire du baptême] et de la consignation [du saint-chrême] ». Gélase pouvait dès lors affirmer de manière péremptoire que ce n'est pas le territoire qui fait la paroisse ou le diocèse, mais bien l'assemblée des fidèles ${ }^{15}$. Un recueil de la chancellerie pontificale, le Liber diurnus, utilise, au viI ${ }^{\mathrm{e}}$ siècle (ou au vIII ${ }^{\mathrm{e}}$ siècle), dans un formulaire relatif aux dédicaces d'églises, l'expression circumiecta plebs, le "peuple se trouvant tout autour", pour décrire l'affluence, le jour de la cérémonie de dédicace, des fidèles dépendant d'une église (et résidant donc autour de celle-ci) ${ }^{16}$, suggérant ainsi une sorte de projection spatiale de la plebs, c'est-à-dire de l'assemblée des fidèles, disposée de manière presque 
circulaire autour du point fixe que constituait le lieu de culte. Pour les évêques carolingiens, chaque "église" ou "paroisse " se voyait en tout cas attribuer une plebs ${ }^{17}$.

Formant des plebes, les fidèles se trouvaient en même temps réunis dans des plebes, ainsi qu'on le disait en de nombreuses régions, comme l'Afrique d'Augustin ${ }^{18}$, la Provence et, jusqu'à une date tardive, l'Italie centrale et septentrionale, ou encore la Bretagne (sous la forme plou). Comme l'indique le mot, la dimension territoriale n'est pas première dans la plebs du haut Moyen Âge : utilisé tout d'abord pour qualifier un rassemblement de fidèles, plebs se mit ensuite, de manière progressive mais non exclusive, à désigner l'église dont dépendaient ces fidèles, comme dans ce concile romain de 826 qui évoque des plebes baptismales (c'est-à-dire des églises baptismales) et fait allusion au populus d'une plebs (c'est-à-dire aux fidèles d'une église) ou dans ce capitulaire de 876, où les fidèles résidant dans les campagnes sont appelés à se rendre régulièrement in plebe, «dans leur église " ${ }^{19}$. Ce n'est qu'en un troisième temps que le mot plebs, qui était donc passé du sens de "fidèles» à celui d'« église», en vint également à qualifier le ressort ou territoire d'une église ${ }^{20}$.

11 Ainsi, pendant longtemps, la réunion des fidèles fit et définit la plebs ou la paroisse, de même que l'autorité épiscopale exercée sur un certain nombre de plebes faisait et définissait le diocèse. Dans cette perspective, l'évêque devait se déplacer pour rendre visite aux groupes de fidèles et aux prêtres relevant de son gouvernement. La référence à cette relation personnelle et au déplacement qu'elle exigeait - d'une assemblée de fidèles à l'autre - représente l'un des usages les plus fréquents du terme parochia dans le haut Moyen Âge. Dans tous les documents, normatifs et narratifs, le déplacement de l'évêque est envisagé, depuis le $\mathrm{vl}^{\mathrm{e}}$ siècle au moins, comme une circulation ou circumambulation au sein de la paroisse (au singulier) ou parmi les paroisses (au pluriel) : circumire / circuire parochiam ou per parochias ${ }^{21}$. Cette circulation épiscopale, recommandée par les conciles gaulois, par des papes, comme Grégoire le Grand, par les synodes et les capitulaires carolingiens et bien d'autres textes, était justifiée par la nécessité de la prédication et de l'enseignement, par la volonté de procéder à la confirmation et de surveiller le populus ou la plebs ${ }^{22}$.

12 Le mot parochia fut donc utilisé de manière privilégiée pour signifier le lien qui devait se nouer entre des groupes de fidèles et l'autorité ecclésiastique ; ce lien, qui supposait des déplacements, se cristallisait autour des lieux de culte. L'association fréquente du mot parochia à l'idée de « circuit » et de « circumambulation » n'est sans doute pas sans rapport avec les «circuits» processionnels effectués, depuis l'Antiquité, par des pèlerins dont les parcours mettaient en valeur certains lieux de culte ${ }^{23}$. Elle renvoie également au rite de consécration des églises qui comportait, à partir des $\mathrm{VII}^{\mathrm{e}}-\mathrm{VIII}^{\mathrm{e}}$ siècles, la réalisation d'une procession, qualifiée de circuitus, autour des édifices ecclésiaux ${ }^{24}$ : de même que le « circuit » effectué par un prélat consécrateur instituait véritablement les églises; paroisses et diocèses prenaient consistance par la circulation d'un évêque.

«Terminum » et paroissiens

Dans l'Occident carolingien, l'institution ecclésiale investit nombre de pratiques et d'institutions sociales qui lui étaient jusqu'alors demeurées extérieures. Afin de subvenir aux besoins d'une Église qui se voulait désormais omniprésente, les autorités affirmèrent le caractère obligatoire de la dîme (capitulaire de Herstal en 779, synode de Francfort en 794, synode de Salz en 803-804, capitulaire d'Aix-la-Chapelle en 813); de ce 
fait, des zones de prélèvement furent rattachées aux lieux de culte. Le capitulaire ecclésiastique de 810-813, repris dans les collections canoniques postérieures, évoque très explicitement le "territoire" (terminum) dont toute église devait désormais disposer et à l'intérieur duquel il lui fallait percevoir « la dîme des uille » (ce qui signifie que plusieurs uille pouvaient dépendre d'une église, ainsi que le confirme d'ailleurs, à la même époque, un formulaire relatif aux constructions d'églises) ${ }^{25}$. Hincmar de Reims répète cette prescription en $857-858$, dans sa collection-traité Sur les églises et les chapelles, avant de rappeler qu'il est "impie», d'après l'« Écriture divine», de "déplacer les limites établies par nos pères (terminos a patribus constitutos) ${ }^{26}$ : ce faisant, l'archevêque de Reims faisait clairement le lien - peut-être pour la première fois - entre le territoire dépendant de chaque église (terminum) et l'injonction à ne pas déplacer ses limites (terminos); ainsi les passages de l'Écriture concernant les bornes à ne pas franchir se trouvaient-ils résolument interprétés en un sens territorial.

Ces territoires ecclésiaux, auxquels faisait allusion un nombre croissant de documents normatifs à partir du début $\mathrm{du} \mathrm{Ix}^{\mathrm{e}}$ siècle, furent parfois qualifiés de "paroisses ». Hincmar de Reims, qui interdit à maintes reprises les "divisions " de "paroisses ", ainsi que l'ont montré Philippe Depreux et Cécile Treffort ${ }^{27}$, recourt notamment à un texte, placé sous le nom d'un pape Denys (III ${ }^{e}$ siècle), qui prescrit le respect de la répartition des "paroisses" et des "cimetières" (parochias et cimiteria) entre les prêtres. Selon ce texte, qui est en réalité une forgerie reprise au recueil des Fausses Décrétales (rassemblées peut-être dès 834-835), aucun prêtre ne devait transgresser " les limites de la paroisse ou le droit d'un autre » (alterius parrochiae terminos aut ius ${ }^{28}$ ): limites et paroisses sont ici explicitement liées.

Ainsi le nouvel ordre carolingien paraît-il avoir favorisé l'image d'une Église constituée d'aires territoriales, quelquefois dénommées " paroisses ", dont les habitants furent dès lors qualifiés de "paroissiens » (parochiani). Le capitulaire déjà mentionné de 810-813, puis d'autres textes épiscopaux et conciliaires affirmèrent le caractère infrangible du lien qui unissait le prêtre d'un lieu de culte à ses paroissiens : aucun célébrant ne devait solliciter, ni même accepter dans son église le « paroissien d'un autre [prêtre] » (alterius parochianum), sauf si celui-ci se trouvait en voyage, c'est-à-dire éloigné de l'église et du prêtre dont il dépendait habituellement ${ }^{29}$. Le terme même de "paroissien" (parochianus), qui ne semble pas attesté avant le $\mathrm{IX}$ siècle et dont la forme adjectivale pouvait alors qualifier le prêtre responsable d'une église ou l'évêque gouvernant un diocèse, entra progressivement dans l'usage, sous forme de substantif, pour désigner de manière à peu près exclusive et plurielle les fidèles ${ }^{30}$.

Dans un premier temps, ceux-ci paraissent avoir été considérés comme " paroissiens " par rapport à la figure d'autorité qu'incarnait leur prêtre plutôt qu'en raison de leur résidence dans une " paroisse ». Le rite de la pénitence joua sans doute un rôle essentiel dans l'établissement d'une telle relation entre le prêtre et ses "paroissiens ». Les conciles s'inquiètent, en effet, de ce que des "paroissiens " puissent se soustraire à la confession, et soulèvent même le cas des fidèles vivant en plusieurs lieux et relevant donc de différentes "paroisses »: il était alors demandé à leurs "voisins » de vérifier $s^{\prime}$ ils se confessaient régulièrement ${ }^{31}$. Dans un second temps, à des moments variables selon la typologie et la géographie des documents, mais en tout cas entre le $\mathrm{x}^{\mathrm{e}}$ et le $\mathrm{XII}^{\mathrm{e}}$ siècle, le mot parochiani (le plus souvent au pluriel) qualifia les fidèles en tant qu'ils vivaient ou habitaient dans le ressort d'une église et s'inscrivaient au sein d'un territoire. Les rédacteurs de chartes parlent alors des "paroissiens d'une 
uilla ( parochiani uillae), des «habitants et paroissiens du voisinage » (incole et parrochiani ibi adiacentes), des "paroissiens qui habitent ou habiteront » (parrochiani qui habitant uel habitabunt) en un lieu donné, et tel seigneur peut faire édifier une église " avec tous ses vilains et ses paroissiens » (cum omnibus uillanis et parrochianis suis) ${ }^{32}$. Au début du XII ${ }^{\mathrm{e}}$ siècle, un acte de l'archevêque de Pise évoque même les " paroissiens qui sont compris dans le territoire » d'une église plébane : parrochiani qui infra territorium predicte plebis continentur ${ }^{33}$.

17 La paroisse se faisait territoire. Quelques documents mentionnent le fait d'« habiter» ou de «résider dans une paroisse» ${ }^{34}$. Il reste que le découpage de territoires paroissiaux est attesté plus précocement pour certaines régions d'Occident que pour d'autres. En Catalogne, une abondante production écrite, en particulier des actes de dotation et de consécration d'églises, conservés parfois sous forme d'originaux, livrent très tôt de précieuses indications sur l'appréhension territoriale des cellules de base de la société chrétienne. À partir du milieu du $\mathrm{x}^{\mathrm{e}}$ siècle, en effet, ces actes consignent par écrit la constitution de "paroisses ", leur dotation en terres (toujours situées, souvent délimitées), ainsi qu'en «dîmes, prémices et oblations» dues par des fidèles "travaillant dans ces territoires " ${ }^{35}$. Lorsque l'évêque et parfois le comte "donnent " ou " concèdent " une " paroisse ", cela signifie très clairement, dans le vocabulaire de ces actes, qu'ils délimitent et attribuent un territoire à un lieu de culte: dedimus terminus ad affrontationes ad ipsa ecclesia ${ }^{36}$, ou : concesserunt ad iam dictam ecclesiam termini parrochie sue ${ }^{37}$. Les actes consignent alors par écrit, avec précision, les « limites paroissiales $"{ }^{38}$.

18 L'assimilation de la paroisse à un territoire paraît dans certains cas totale, comme dans cet acte marseillais qui évoque, en 1096, les possessions des moines de Saint-Victor dans une «paroisse » qui coïncide avec le «territoire d'un château»: in eandem parrochia uel in territorio iamdicti castelli ${ }^{39}$. Il y aurait beaucoup à dire sur cette formule, qui renvoie notamment à la question des églises paroissiales fondées par les maitres de châteaux et au recouvrement éventuel entre le territoire castral et le ressort paroissial ${ }^{40}$; pour notre propos, je note seulement que la parrochia est un territorium, son ressort correspondant à l'aire de domination d'un site fortifié.

Territorialisation, distance et voisinage

19 Plusieurs éléments favorisèrent le processus de territorialisation des églises locales, qu'il convient donc d'envisager dans une longue durée : $\mathrm{IX}^{\mathrm{e}}$-XII ${ }^{\mathrm{e}}$ siècles, ainsi que paraît le confirmer l'usage des termes parochia et parochiani. Nous avons vu que les premiers textes (carolingiens) mentionnant le terminum des églises portent sur la dîme. Imposé en principe à tous, le prélèvement même de la dîme induit l'existence d'un territoire, $\mathrm{du}$ fait que tous les habitants dépendant d'une église se trouvent soumis au prélèvement : le territoire est donc l'espace où vivent les fidèles qui doivent s'acquitter de la dîme ${ }^{41}$. Tous n'y étaient cependant pas assujettis de manière identique, et il fallut plusieurs siècles avant que les ecclésiastiques ne s'emploient à percevoir effectivement la dîme.

20 À en juger par les directives imposées à partir du $\mathrm{IX}^{\mathrm{e}}$ siècle, la pratique de la confession contribua également à attacher les fidèles à une église locale, les habitants qui en dépendaient se voyant d'ailleurs chargés, en bons "voisins ", d'une sorte de contrôle mutuel. Mais c'est surtout l'obligation pour les populations de baptiser leurs enfants et d'inhumer leurs défunts en un lieu proche de leur résidence qui favorisa la formation de zones paroissiales. Dans une lettre adressée en 853 à son confrère Thibaud de 
Langres, l'archevêque Amolon de Lyon définit la "paroisse» comme le lieu où les fidèles sont baptisés, communient, se confessent, payent la dîme et sont ensevelis ${ }^{42}$, ce qui supposait donc qu'ils puissent se rendre sans trop de difficultés en ce lieu.

Or, c'est précisément à partir $\mathrm{du} \mathrm{IX}^{\mathrm{e}}$ siècle que les autorités ecclésiastiques se préoccupèrent de la possibilité pour toute collectivité d'habitants de rejoindre un lieu de culte, notamment pour y ensevelir les défunts - à cette époque où les sépultures commençaient à se concentrer autour des églises et où les prêtres se voyaient chargés de veiller à l'inhumation de leurs "paroissiens ${ }^{43}$. Une sorte de distance critique maximale entre le domicile des fidèles et l'église qui devait les accueillir fut dès lors établie (quatre ou cinq milles, soit une vingtaine de kilomètres, selon le concile de Tribur en 895, une distance qui sera presque réduite de moitié dans les recommandations des conciles $\mathrm{du}$ xIII ${ }^{\mathrm{e}}$ siècle) ${ }^{44}$. Textes normatifs et chartes soulignèrent donc la nécessité pour les fidèles d'avoir accès à un lieu de culte proche de leur résidence afin d'y ensevelir les corps de leurs défunts, et l'on en vint même à désigner les fidèles d'une église comme les « paroissiens de [son] cimetière » (parochiani cimiterii) ${ }^{45}$. Parallèlement, la nécessité de recevoir en un lieu proche le «sacrement de la régénération ", c'est-à-dire le baptême, moins fréquemment évoquée, se rencontre toutefois jusqu'au XII ${ }^{\text {e }}$ siècle, en particulier en Italie ${ }^{46}$. De toute manière, le principe de la proximité des églises locales, quelle qu'en fût la raison (le paiement de la dîme, la confession, le baptême ou la sépulture), renvoyait à l'idée d'une nécessaire circulation (celle des fidèles en l'occurrence) de leur lieu de vie à un lieu de culte. La possibilité d'une telle circulation représentait une sorte de préliminaire ou de condition à l'établissement d'aires territoriales. La formation d'un territoire paroissial dépendait, idéalement, de la capacité des fidèles à se rendre auprès d'un prêtre et dans une église qui leur fussent propres, un peu de la même manière que la circulation de l'évêque d'un groupe de fidèles à l'autre faisait le diocèse.

La paroisse qui se mit en place entre le $\mathrm{IX}^{\mathrm{e}}$ et le $\mathrm{XII}^{\mathrm{e}}$ siècle doit donc être définie comme une structure spatiale assurant aux populations des lieux de culte de proximité. C'est du reste la raison pour laquelle le lien unissant un prêtre et ses paroissiens ne se dénouait que lorsque ces derniers se trouvaient éloignés de leur domicile, sur la route (in itinere): en somme, il n'y avait de paroissien que d'un prêtre ou d'une église spatialement proche. Une même logique était sous-jacente à la définition des devoirs pastoraux du prêtre et de l'évêque: de même que le premier ne devait "pas abandonner ses fidèles » (non plebem suam deserere, selon Hincmar de Reims), le second ne devait pas quitter sa " cité », selon le concile de Paris de 829, pour « fréquenter des lieux plus éloignés ", négligeant ainsi la prédication qu'il devait assurer in plebibus sans doute « dans ses églises » plutôt qu' « à ses fidèles » ${ }^{47}$. Les considérations relatives à la distance, au voisinage et à la proximité renvoyaient à l'étymologie même du mot

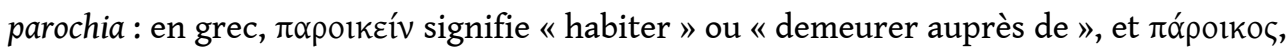
«voisin» ou "proche de». Les auteurs du Moyen Âge n'ignoraient pas cette étymologie, qu'ils rappellent: la parochia est la "maison proche» (adiacens domus), étant entendu qu'il s'agissait d'une «maison de Dieu » (id est domus Dei) ${ }^{48}$.

Tandis que le terme parochia (au féminin singulier) se mettait à qualifier un territoire, il fut également utilisé (au neutre pluriel) pour désigner les revenus paroissiaux. Le lien est évident entre les deux réalités: l'organisation et le contrôle d'un territoire se traduisaient par la perception de revenus (dîmes, prémices, droits funéraires et offrandes diverses) sur ce territoire. La même évolution est perceptible pour 
cimiterium/cimiteria qui désignent, à partir des $\mathrm{x}^{\mathrm{e}}$ et $\mathrm{xI}^{\mathrm{e}}$ siècles, l'espace funéraire collectif désormais attenant à l'église (au singulier) et les droits de sépulture perçus par les clercs (au pluriel). En d'autres termes, les dimensions territoriale et fiscale de la parochia constituent les deux aspects d'un même phénomène. Et comme l'écrit Martial Staub, en liant étroitement les revenus ecclésiaux à l'administration des sacrements et des rites, l'institution de la dîme, des droits de sépulture et des autres offrandes « revenait à mettre entre parenthèses le groupement » (c'est-à-dire le populus, la plebs), " auquel finit d'ailleurs effectivement par se substituer un ressort » (c'est-à-dire la parochia comme territoire) ${ }^{49}$.

La « parochia » comme territoire

Les partisans de la réforme de l'Église ne modifièrent pas sensiblement, au XI ${ }^{\mathrm{e}}$ siècle, la conception que les clercs se faisaient de la parochia, le terme continuant du reste à être utilisé pour désigner aussi ce que nous appellerions un diocèse. Du moins les églises locales se trouvèrent-elles assimilées, comme tous les biens ecclésiastiques, à des res sacrae inaliénables, telles que les définissait le droit romain dans lequel puisaient les réformateurs. Les papes, Grégoire VII en particulier, puis ses successeurs, se préoccupèrent toutefois davantage des diocèses et des provinces ecclésiastiques que des paroisses, affirmant notamment leur prérogative, en tant que successeurs de Pierre, dans la création, la modification ou la suppression de ces plus vastes circonscriptions ${ }^{50}$. Par ailleurs, tout usage métaphorique de la référence à la limite ne disparut pas: l'intervention de Grégoire VII dans les élections épiscopales est par exemple dénoncée dans les libelles polémiques anti-grégoriens comme une «transgression » des « bornes » ou une « invasion de paroisse » ${ }^{51}$.

Quoi qu'il en soit, le processus de territorialisation était à l'œuvre. La multiplication des conflits de «juridiction» et de "limites» entre paroisses, que tous les historiens constatent dans leur documentation, parfois dès le $\mathrm{XI}^{\mathrm{e}}$ siècle, et plus généralement au cours des $\mathrm{XII}^{\mathrm{e}}$ et XIII ${ }^{\mathrm{e}}$ siècles, est un indice de l'achèvement de ce processus : la formation d'unités territoriales compactes, continues et articulées les unes aux autres nécessitait, en effet, des ajustements et des délimitations, engendrant de multiples litiges entre ces ensembles désormais contigus et exclusifs ${ }^{52}$. L'image de la réalité paroissiale qui s'imposa alors fut celle d'une église qui rayonnait comme un candélabre illuminant la "paroisse se trouvant tout autour", selon le mot du chanoine régulier Gerhoh de Reichersberg vers $1130^{53}$ : cette parochia circumiacens se substituait en quelque sorte à la circumiecta plebs du haut Moyen Âge.

Le terme parochia est bien attesté, au milieu du XII ${ }^{e}$ siècle, dans le Décret de Gratien : 96 attestations du substantif, auxquelles il faut ajouter 21 occurrences des adjectifs parochialis, parochianus ou parochitanus (généralement associés au mot ecclesia) et 14 attestations du substantif parochianus, - $i$ (pour désigner les fidèles). Certes, le Décret charrie-t-il (en la réorganisant) toute la tradition canonique du haut Moyen Âge, mais lorsque Gratien prend personnellement la parole, il investit parochia d'un sens nettement territorial, comme dans ce dictum où la "paroisse" désigne la zone dépendant d'une "église paroissiale ", de la même manière que le "diocèse » est la zone assignée à une "église baptismale ": Constat unamquamque baptismalem ecclesiam habere diocesim sibi legitime assignatam, sicut et parrochiales ecclesie habent parrochias distributas ${ }^{54}$. Commentant ce passage, le grand canoniste Huguccio explique d'ailleurs, à la fin du XII ${ }^{\mathrm{e}}$ siècle, que la paroisse est, comme le diocèse, le « territoire " d'une église ${ }^{55}$. Dans un autre dictum, Gratien avait envisagé la possibilité pour un évêque de diviser 
une paroisse en deux ou au contraire d'unir deux paroisses, division et union renvoyant aussi à une perception territoriale de la réalité paroissiale ${ }^{56}$.

Une telle perception est encore présente dans le Décret lorsque Gratien affirme que le fidèle doit s'acquitter de la dîme à l'église du lieu où sont établies ses possessions foncières (et non à l'église de son choix, ni même à l'église où il recevrait les sacrements) ${ }^{57}$ : cette règle rompt avec la forte relation personnelle, plutôt que territoriale, qui avait pendant longtemps caractérisé les liens entre les fidèles et leur église. Dans la seconde moitié du xII ${ }^{\mathrm{e}}$ siècle, les décrétistes ont poursuivi dans la même voie. Selon Simon de Bisignano, par exemple, les dîmes doivent être prélevées en fonction des possessions foncières (ratione prediorum) et non des personnes (non ratione personarum). Ceci pouvait conduire à minorer l'importance du domicile d'un fidèle au profit de la localisation de ses terres ${ }^{58}$, et en allant jusqu'à bout de cette logique, à concevoir un territoire paroissial sans fidèles mais constitué de biens-fonds alimentant la dîme. Un autre canoniste, Jean de Faenza, remarque que la dîme n'a pas toujours été prélevée selon les mêmes modalités : dans un passé plus ou moins lointain, lorsque les " paroisses » n'étaient pas " distinguées », quelle que fût l'église dans laquelle un fidèle recevait les sacrements, c'est à cette église qu'il devait donner la dîme ; ce n'est que par la suite, après que l'on eut procédé à la " délimitation » des "diocèses ", que la dîme ne fut plus perçue en fonction des personnes mais des biens-fonds, de sorte qu'elle revint à l'église dans le territoire de laquelle se trouvaient les biens du fidèle ${ }^{59}$.

Les «paroissiens » ne sont pas absents du Décret: Gratien rappelle à plusieurs reprises qu'aucun évêque ou prêtre ne peut attirer le "paroissien d'un autre [évêque ou prêtre] ». Celui-ci tend d'ailleurs à être considéré comme le "paroissien d'une autre église " plutôt que comme celui d'un évêque ou d'un prêtre ${ }^{60}$. Gratien précise en outre que si un tel détournement de paroissien se produisait, il conviendrait, après enquête, de renvoyer le fidèle « dans sa paroisse ${ }^{61}$.

Cependant, lorsque Gratien relit ou réinterprète en un sens territorial les textes anciens relatifs à la parochia, il les applique le plus souvent au cas du diocèse, c'est-àdire au territoire de l'évêque, plutôt qu'à la paroisse ${ }^{62}$. Du reste, pas plus que ceux de l'âge grégorien, les canonistes $\mathrm{du} \mathrm{xII}^{\mathrm{e}}$ siècle ne donnent de définition claire de la «paroisse ». Pour disposer d'une telle définition, ainsi que d'une réflexion plus précise sur le territoire et les limites de l'institution paroissiale, il faut attendre le XIII ${ }^{\mathrm{e}}$ siècle. C'est, en effet, Henri de Suse (dit Hostiensis) qui, vers 1250, dans sa Somme Dorée, note que les principes valant pour la répartition des diocèses (énoncés par Gratien et ses successeurs) concernent tout aussi bien les paroisses. Ce grand canoniste est aussi le premier à définir explicitement la parochia, qu'il envisage comme un cadre de vie pour les fidèles, correspondant à un espace strictement délimité et sanctionné par un «droit » : «Qu'est-ce qu'une paroisse ? Un lieu dans lequel vit un peuple attribué à une église, délimité par des frontières sûres; on le considère comme paroisse dans la mesure où s'y étend le droit spirituel de l'église.» La dimension territoriale est essentielle : « Il ne peut y avoir, sur un même territoire, plusieurs églises baptismales » (ici, l'église baptismale est synonyme d'église paroissiale) et dès lors, « il faut répartir et délimiter les églises baptismales ». Mentionnant comme ses devanciers la Pseudodécrétale du pape Denys, le canoniste précise qu'au sein de l'Église, l'usage de délimiter un territoire a commencé sous le gouvernement de ce pape ${ }^{63}$.

Les réflexions des canonistes sur la paroisse comme territoire s'accompagnèrent de remarques sur la manière de repérer les limites territoriales lorsque celles-ci n'étaient 
pas évidentes. Le pape Honorius III avait recommandé de reconstituer les limites en se servant de documents anciens ou en se fiant à des témoins et à la rumeur publique ${ }^{64}$. Les canonistes évoquent aussi les monuments et les bornes qui peuvent témoigner des limites, mais Henri de Suse fait remarquer qu'il est difficile de retrouver ces monuments anciens ${ }^{65}$.

Le «paroissien » se définit «en fonction de son domicile» (ratione domicilii), écrit encore Henri de Suse qui combine ainsi les dimensions territoriale et personnelle de la paroisse, celle-ci étant faite de fidèles insérés (ou enserrés) dans un territoire. Il n'est qu'un « voyageur » qui puisse être considéré comme " paroissien » de toute église dans laquelle il se rendrait ${ }^{66}$. Quant au «droit paroissial », il se fonde sur la " cure des âmes des paroissiens " et consiste "en dîmes et prémices", "en cimetière et droit de sépulture ", "dans le fait d'écouter les pénitents», "en revenus de provenances diverses ", " en oblations " ${ }^{67}$. Certains de ces droits ancrent territorialement (la dîme, la sépulture), d'autres renvoient à des relations personnelles (cure des âmes, confession). En rappelant que l'appartenance paroissiale se fondait sur la confession régulière du fidèle à son "propre prêtre" (canon 21), lequel ne pouvait recevoir plusieurs « églises paroissiales " (canon 29) ${ }^{68}$, le concile de Latran IV, en 1215, avait préparé une telle conception de la paroisse, délimitée territorialement mais toujours fondée sur des relations personnelles.

\section{NOTES}

1. Modèle du genre : P. Imbart de la Tour, Les Origines religieuses de la France. Les paroisses rurales du IV e au XI e siècle, Paris, 1900, ainsi que l'article de M. Chaume, « Le mode de constitution et de délimitation des paroisses rurales aux temps mérovingiens et carolingiens ", Revue Mabillon 27, 106, 1937, p. 61-73.

2. Outre les contributions réunies dans ce numéro de Médiévales, cf. par exemple J. Avril, « La "paroisse" dans la France de l'an Mil », dans Le Roi de France et son royaume autour de l'an mil. Actes du Colloque Hugues Capet, Paris-Senlis, 22-25 juin 1987, M. Parisse, X. Barral i Altet éd., Paris, 1992, p. 203-218 (ici p. 203), et A. Dierkens, « Les paroisses rurales dans le nord de la Gaule pendant le haut Moyen Âge. État de la question et remarques critiques ", dans La Paroisse en questions. Des origines à la fin de l'Ancien Régime, Athis-Mons-Saint-Ghislain, 1998, p. 21-47 (ici p. 24).

3. Cf. Alle origini della parrocchia rurale (IV-VIII sec.). Actes du Colloque de Rome, 19 mars 1998, Ph. Pergola, P.M. Barbini éd., Vatican, 1999, ainsi que Aux origines de la paroisse rurale en Gaule méridionale, IV $V^{e}-I X^{e}$ siècles. Actes du Colloque de Toulouse, 21-23 mars 2003, C. Delaplace éd., Toulouse, 2005.

4. Ch. Delaplace, « Les origines des églises rurales ( $\mathrm{v}^{\mathrm{e}}-\mathrm{vi}{ }^{\mathrm{e}}$ siècles). À propos d'une formule de Grégoire de Tours », Histoire et Sociétés Rurales, 18, 2002, p. 11-40 (ici p. 27). 5. «Placuit obseruari ut si quis episcopus in ius fratris sui suam conatus fuerit inserere potestatem ut aut dioceses alienas transgrediendo terminos a patribus constitutos peruadat » (CC SL, 148, p. 146). Voir aussi le concile de Clermont en 535 (CC SL, 148A, p. 107). 
6. Cf. Prv 22, 28 (« ne transgrediaris terminos antiquos quos posuerunt patres tui »), ainsi que Dt 19, 14.

7. On la rencontre notamment, à l'époque carolingienne, dans les Libri carolini et, au moment de la réforme grégorienne, dans les libelles polémiques.

8. Ma contribution se fonde sur les ressources des bases de données textuelles disponibles (CLCLT-5, MGH-5, PL et chartes originales de l'Artem). Je remercie BenoîtMichel Tock qui m'a donné accès à la base de l'Artem.

9. Voir en particulier la longue notice consacrée à « Parochia » dans le plus riche et le plus récent de ces dictionnaires, le Novum Glossarium Mediae Latinitatis, J. Monfrin dir., fasc. Pars-Passerulus, par A.-M. Bautier et M. Duchet-Suchaux, Copenhague, 1989, col. 380-395.

10. Par exemple dans une chaîne de textes provençaux, en commençant par le concile d'Agde de 506 et surtout celui de Vaison en $529:$ «... nobis placuit, ut non solum in ciuitatibus sed etiam in omnibus parrociis uerbum faciendi daremus presbyteris potestatem » (CC SL, 148A, p. 78-79). Le texte est repris dans un sermon de Césaire d'Arles (I, 12) et, plus tard, au concile d'Arles de 813 (MGH Concilia II, 1, p. 251).

11. Attestations dans une lettre (VII, 6) de Sidoine Apollinaire ( $†$ vers 490) et dans les capitulaires (cinq occurrences) de l'archevêque Hincmar de Reims ( $†$ 882).

12. Voir par exemple le concile d'Agde en 506, c. 21 (CC SL, 148, p. 202) et celui d'Epaone en 517, c. 25 : « Sanctorum reliquiae in oratoriis uillarebus non ponantur, nisi forsitan clericus cuiuscumque parrochiae uicinus esse contingat, qui sacris cinerebus psallendi frequentia famulentur » (CC SL, 148A, p. 30).

13. V. Saxer, « Les paroisses rurales de France avant le ix ${ }^{\mathrm{e}}$ siècle : peuplement, évangélisation, organisation ", Les Cahiers de Saint-Michel de Cuxà, 30, 1999, p. 5-41 (ici p. 10). V. Saxer note que $c^{\prime}$ est vers le vii ${ }^{\mathrm{e}}$ siècle que les deux mots tendent vers le sens actuel. Il n'en reste pas moins que l'on rencontre encore, ainsi qu'on vient de le dire, des parochiae-diocèses aux xi ${ }^{\mathrm{e}}$ et xii ${ }^{\mathrm{e}}$ siècles. Cf. aussi Ch. Delaplace, « Les origines des églises rurales... ", p. 17.

14. Concile d'Agde (506), col. $21:$ «... parrocias, in quibus legitimus est ordinariusque conuentus... »(CC SL, 148, p. 202).

15. « Nulla igitur praesumptione statum paroeciarum, qui perpetuae aetatis firmitate durauit, patimur immutari [...]» Un peu plus loin : « ... ex qua semper ad regenerationem atque consignationem plebs deuota conuenit. Territorium etiam non facere diœcesim olim noscitur ordinatum » (fragmentum 17, éd. A. Thiel, Epistolae romanorum pontificum genuinae, t. 1, Brannsberg, 1868, p. 492-493).

16. Liber diurnus Romanorum pontificum, éd. T. VON SICKEL, Vienne, 1889, nº 20, p. 15. 17. Cf. la lettre, datée de 853, de l'archevêque Amolon de Lyon : « Unaquaeque plebs in paroechiis et ecclesiis, quibus attributa est, quieta consistat » (PL 116, 82).

18. Cf. S. Lancel, « À propos des nouvelles lettres de saint Augustin et de la Conférence de Carthage de 411 : Cathedra, Diocesis, Ecclesia, Parochia, Plebs, Populus, Sedes. Remarques sur le vocabulaire des communautés chrétiennes d'Afrique du Nord au début du v $\mathrm{v}^{\mathrm{e}}$ siècle ", Revue d'Histoire Ecclésiastique, 77, 1982, p. 446-454.

19. Pour les deux attestations de plebs-église dans le concile romain de $826: M G H$ Concilia II/1, p. 570 et 577 . Le même concile utilise également plebs au sens de fidèles : «... absentia episcopi plerumque fit calamitas plebis » (p. 570). Plebs-église également dans le capitulaire de 876, donné à Pavie, sous le règne de Charles le Chauve (MGH Capitularia regum Francorum II, p. 102 : « ecclesias baptismales, quas plebes appellant »). Un autre capitulaire (Ravenne, 898) évoque les « plebes aecclesiasticae » (p. 110). 
20. Cette évolution en trois temps (fidèles/église/territoire ecclésial) est donc plus complexe que ne le laissent penser les travaux de P. Aebischer, «La diffusion de plebsparoisse dans l'espace et dans le temps ", Revue de linguistique romane, 28, 1964, p. 143-165 (fort précieux), I. Mazzini, « La terminologia della ripartizione territoriale ecclesiastica nei testi conciliari latini dei secoli iv e v ", Studi urbinati di scienze giuridiche, politiche ed economiche, n.s. $\mathrm{n}^{\circ} 27$ - a. 43, 1974-1975, p. 233-266, et L. Pellegrini, « Plebs et populus in ambito rurale nell'Italia altomedioevale ", dans Società, istituzioni, spiritualità. Studi in onore di C. Violante, 2 vol., Spolète, 1994, p. 599-632, qui partent d'une opposition simple plebs-fidèles/plebs-territoire et s'interrogent sur le passage d'un sens à l'autre. 21. Je ne peux donner ici les dizaines d'occurrences du « circuit » de l'évêque dans sa (ou ses) «paroisse(s) » (formule que relève la notice « Parochia » du Novum Glossarium Mediae Latinitatis, col. 383 et passim). Je prépare une étude sur la notion de " circuitus » dans l'Occident médiéval.

22. Selon un concile de 747 : «Statuimus, ut singulis annis unusquisque episcopus parrochiam suam sollicite circumeat, populum confirmare et plebes docere et inuestigare... » (MGH Concilia II/1, p. 47). Un capitulaire du maire du palais Carloman, en 742, évoquait déjà la « circulation » de l'évêque parmi les fidèles pour

la confirmation (MGH Capitularia regum Francorum I, p. 25). Et le capitulaire de 810-813 prescrit « ut unusquisque episcopus interim circumeat parrochiam suam docendo et ammonendo » (MGH Capitularia regum Francorum I, p. 174).

23. V. Fiocchi Nicolai, «Sacra martyrum loca circuire : percorsi di visita dei pellegrini nei sanctuari martiriali del suburbio romano ", dans L. Pani Ermini dir., Christiana loca. Lo spazio cristiano nella Roma del primo millennio, Rome, 2000, p. 221-230.

24. Voir notamment les premiers rituels : Ordo quomodo ecclesia debeat dedicari ( $=$ ordo 41, éd. M. Andrieu, Les Ordines Romani du haut Moyen Âge, t. 4, Louvain, 1961, p. 339-347) et Ordo quomodo in sancta romana ecclesia reliquiae conduntur (= ordo 42, éd. M. Andrieu, Les Ordines Romani..., p. 397-402).

25. Capitulaire de 810-813, col. $10:$ « Ut terminum habeat unaquaeque aecclesia, de quibus uillis decimas recipiat » (MGH Capitularia regum Francorum I, p. 178). Cette prescription est reprise dans la collection des capitulaires d'Anségise (I, 149 : de termino ecclesiarum) en 827, MGH Capitularia regum Francorum, n.s. I, p. 412. Selon la formule de " cessio ad ecclesiam a nouo aedificatam », datée de 808, plusieurs uille sont de fait rattachées à une église (Formulae Senonenses recentiores, col. 12, MGH Formulae merowingici et karolini aevi, p. 217).

26. Hincmar de Reims, Collectio de ecclesiis et capellis, éd. M. STRATMANN, MGH Fontes Iuris Germanici Antiqui in usum scholarum, 14, 1990, p. 73. La collection-traité d'Hincmar me paraît représenter la première traduction idéologique d'un lent processus de territorialisation de l'Église : M. Lauwers, «Circuit, cimetière, paroisse. Réflexions sur l'ancrage ecclésial des sites d'habitat (vii ${ }^{\mathrm{e}}$-xiiie siècles) », dans Autour du «village » entre Seine et Rhin (IV ${ }^{e}$-XIII ${ }^{e}$ siècles). Actes du Colloque de Louvain-la-Neuve, 16-17 mai 2003, sous presse.

27. Ph. Depreux, C. Treffort, «La paroisse dans le De ecclesiis et capellis d'Hincmar de Reims. L'énonciation d'une norme à partir de la pratique ? ", Médiévales 48, 2005, p. 141-148.

28. Hincmar de Reims, Collectio de ecclesiis et capellis, p. 67. Prescription analogue dans le capitulaire pour la Septimanie de Charles le Chauve en 844, c. 7, MGH Capitularia regum Francorum II, p. 257. 
29. Le capitulaire de $810-813$ prescrit « ut nullus presbiter alterius parrochianum nisi in itinere fuerit uel placitum ibi habuerit, ad missam recipiat » (MGH Capitularia regum Francorum I, p. 178). La prescription est reprise en 827 dans la collection d'Anségise, I 147 (MGH Capitularia regum Francorum, n.s. I, p. 511) et dans les Ordines de celebrando concilio. Même recommandation dans les capitulaires des évêques carolingiens Théodulphe d'Orléans (MGH Capitula episcoporum I, p. 112), Raoul de Bourges (ibid., p. 244), Haito de Bâle (p. 214) et, au x ${ }^{\mathrm{e}}$ siècle, Roger de Trêves (II, p. 67) ou encore dans les actes du concile de Trêves (927), c. 5.

30. Hincmar de Reims évoque à deux reprises les « seniores et parrochiani » (Collectio de ecclesiis et capellis, p. 91 et 92). Dans le corpus des chartes originales de l'Artem, le substantif par(r)ochianus apparaît pour la première fois en 1050 et n'entre dans l'usage (avec 36 attestations) que dans le dernier quart du xi ${ }^{\mathrm{e}}$ siècle. Dans ce corpus, le mot concerne toujours les fidèles, jamais les prêtres.

31. Selon le concile de Trêves (927), c. 14 : «Prouideat presbiter studiose, quatinus nullus sit de parochianis suis, cuius confessionem non suscipiat. De his uero, qui in aliquibus uersantur parochiis propter praedia, que habent in locis plurimis, interrogetur a uicinis, si confessionem acceperunt. » Le rôle du prêtre dans la pénitence publique de ses paroissiens, au début du Carême, est aussi évoqué dans le c. 15 (MGH Concilia VI/1, p. 83).

32. Selon plusieurs actes cités dans la notice du Novum Glossarium cit., p. 406.

33. Ceux-ci sont appelés à payer leur dîme à la plebs et à y ensevelir les corps de leurs défunts (acte de 1125, éd. N. Caturegli, Il regesto della Chiesa di Pisa, Rome, 1938, n 298 , p. 193).

34. En 742, Carloman, faisant connaître les décisions des synodes réunis sous l'autorité de Boniface, évoque les prêtres « habitant dans la paroisse ", le terme renvoyant ici à l'espace épiscopal (MGH Capitularia regum Francorum I, p. 25). En 932, ce sont les fidèles résidant dans la paroisse, et non plus les prêtres, qu'évoque le concile de Dingolfing : « unusquisque in unaquaque parochia degens » (MGH Concilia VI/1, p. 124), chacun devant verser, chaque année, un denier à « son prêtre ».

35. Je cite ici une charte originale datée de $948:$ «... cedo ibi decimas et primitias et oblationes fidelium quod infra hos terminos laborauerunt... » (éd. C. Baraut, Les actes de consagracions d'esglésies de l'antic bisbat d'Urgell (segles IX-XII), Urgell, 1986, nº 31, p. 94).

36. Acte de 984, éd. C. Baraut, Les Actes, $n^{\circ} 41$, p. 113.

37. Acte de 984, éd. C. Baraut, Les Actes, $n^{\circ} 42$, p. 114.

38. Acte de 1037, conservé en original : «Sunt autem parochiales termini [...]; quantum iste afrontaciones includunt totum damus in parochiam predicte ecclesie... » (éd. C. Baraut, Les Actes, $n^{\circ} 48$, p. 122).

39. La charte, conservée sous forme originale, est éditée dans B. Guérard, Cartulaire de l'abbaye Saint-Victor de Marseille, Paris, 1857, t. 2, p. 565-566.

40. É. Zadora-Rio, « Construction de châteaux et fondation de paroisses en Anjou aux $\mathrm{xi}^{\mathrm{e}}$-xii ${ }^{\mathrm{e}}$ siècles ", Archéologie Médiévale, 9, 1979, p. 115-123, et, sur le territorium castri en Provence, M.-P. Estienne, Châteaux, villages, terroirs en Baronnies, $\mathrm{X}^{e}-\mathrm{XV} \mathrm{V}^{e}$ siècles, Aix-enProvence, 2004, p. 79-88, 167-238.

41. Cf. J. SEMMLER, «Zehntgebot und Pfarrtermination in karolingischer Zeit », dans Aus Kirche und Reich. Studien zur Theologie, Politik und Recht im Mittelalter. Festschrift für Friedrich Kempf, éd. H. Mordek, Sigmaringen, 1983, p. 33-44, qui surévalue toutefois le processus de territorialisation à l'œuvre dès l'époque carolingienne. 
42. « ... ubi sacrum baptisma accipit, ubi corpus et sanguinem Domini percipit, ubi missarum solemnia audire consueuit, ubi a sacerdote suo poenitentiam de reatu, uisitationem in infirmitate, sepulturam in morte consequitur, ubi etiam decimas et primitias suas offerre praecipitur, ubi filios suos baptismatis gratia initiari gratulatur, ubi uerbum Dei assidue audit » (PL 116, 77-84, ici 82).

43. Cf. Hincmar de Reims, Capitula III, MGH Capitula episcoporum, 2, p. 74.

44. Sur cette question, cf. M. Lauwers, "Circuit, cimetière, paroisse ", loc. cit.

45. Selon l'acte rapportant, en 1098, l'érection de l'église et du cimetière de la Roë, en Mayenne (éd. É. Laurain, « Questions fabriciennes », Bulletin de la Commission historique et archéologique de la Mayenne, 1908, p. 348-349 ; je remercie D. Pichot pour cette référence).

46. Des exemples sont cités, pour les xii ${ }^{\mathrm{e}}$ et xiii ${ }^{\mathrm{e}}$ siècles, par M. Ronzani, "L'organizzazione della cura d'anime (con particolare riguardo alla nascita della pieve di Figline) ", dans “Lontano dalle città". Il Valdarno di Sopra nei secoli XII e XIII , éd. P. Pirillo, sous presse, consultable en ligne sur Reti medievali.

47. Hincmar de Reims, Capitula IV, MGH Capitula episcoporum, 2, p. 81 ; De ordine palatii, éd. Th. Gross et R. Schieffer, MGH Fontes Iuris Germanici Antiqui in usum scholarum, 3, 1980, p. 58 (« episcopi (...) debent (...) non diutius (...) a suis abesse parochiis ») ; concile de Paris, MGH Concilia II/1, p. 627.

48. Selon Eucher de Lyon ( $v^{\mathrm{e}}$ siècle) : « Paroecia, adiacens domus, id est Dei » (Instruction II, 445, CC SL, 66, p. 214), que reprennent Papias (milieu xi ${ }^{\mathrm{e}}$ siècle) et Huguccio (fin xii ${ }^{\mathrm{e}}$ siècle) : «Item a para hec parochiae, adiacens domus domui Dei uel incolatus uel diocesis » (Derivationes, éd. E. Cecchini, Florence, 2004, p. 902).

49. M. Staub, Les Paroisses et la Cité. Nuremberg du XIII siècle à la Réforme, Paris, 2003, p. 19.

50. H.-J. SchмIDT, « Grenzen in der mittelalterlichen Kirche. Ekklesiologie und juristische Konzepte », dans Grenzen und Raumvorstellungen (11.-20. Jh.) - Frontières et conceptions de l'espace (XI ${ }^{e}-X X^{e}$ siècles), G. Marchal éd., Zürich, 1996, p. 137-162 (ici p. 144).

51. M. Lauwers, « Territorium non facere diocesim... Réflexions sur la perception territoriale du diocèse dans l'Occident médiéval ( $v^{\mathrm{e}}$-xiiie siècles) », dans F. Mazel dir., L'Espace du diocèse dans l'Occident médiéval, Rennes, à paraître.

52. Le fait est relevé, avec de légers décalages chronologiques, dans nombre d'études monographiques. Voir, par exemple, récemment, D. Pichot, Le Village éclaté. Habitat et société dans les campagnes de l'Ouest au Moyen Âge, Rennes, 2002, p. 233-235. Il a été récemment étudié de manière précise par $\mathrm{F}$. Hautefeuille, dont on verra la contribution dans ce volume.

53. « Aecclesia uero de sua portione tantum decoris in libris, uestimentis ac ceteris utensilibus accipiat, ut quasi candelabrum in medio collocatum non solum utilitatem per spiritalem illuminationem, sed etiam decorem et exteriorem pulchritudinem circumiacenti parrochiae prebeat » (Gerhoh de Reichersberg, Opusculum de edificio Dei, dans MGH Libelli de lite, 3, p. 195).

54. Décret, IIa pars, C 13, q 1, c 1, dictum ante (F I 717). Ce dictum introduit (et glose) le texte de la Pseudo-décrétale du pape Denys qui évoquait la répartition des « paroisses et cimetières » entre les prêtres.

55. Huguccio, ad Decr. IIa pars, C 13, q 1, c 1, dictum ante : « ... ut parrochia uel diocesis dicatur cuiuslibet ecclesie territorium » (ms Paris, BnF, latin 15396, fo 188ra).

56. Décret, IIa pars, C 16, q 1, c 53, dictum post : F I 778. Quelques décennies plus tard, le théologien parisien Pierre le Chantre évoque la même possibilité (« due ecclesie in 
unam congregantur uel una in duas diuidatur »), puis appelle à ne pas ériger d'autel contre un autel déjà existant, ni une chapelle à l'intérieur de "limites paroissiales " reconnues : « Non erigatur altare contra altare, non fundentur capelle intra limites parrochiales de nouo... » (Verbum adbreuiatum, I, 24, CC CM, 196, p. 203).

57. Décret, IIa pars, C 13 q 1 c 1, dictum post : F 718 (mais il est vrai qu'ici, c'est le terme « diocèse » qui est employé).

58. "Decime enim ratione prediorum non ratione personarum conferuntur. Nam licet possessiones dominus in alia parrochia habeat domicilium et in alia moretur, illis tamen tenetur de prediis dare decimas in quorum parochia sunt predia constituta... » (Simon de Bisignano, ad Decr. IIa pars, C 13, q 2, édition consultable en ligne : http:// www.unifr.ch/cdc/or/SummaIIC13-18).

59. Jean de Faenza, ad Decr. IIa pars, C 13, q 1, c 1, dictum ante : « In priori quidem tempore, nondum distinctis parochiis, dabantur decime personarum dumtaxat contemplatione, scilicet ut in quacumque ecclesia aliquis sue sacramenta salutis percipiet uel diuina officia assidue audisset, ibi omnium suorum bonorum decimas daret. Postquam autem singulorum diocesium limitatio facta est, accessit institutio decimarum intuitu etiam prediorum... » (ms. Paris, BnF, latin 17528, $\mathrm{f}^{\circ} 106 \mathrm{v}^{\circ}$ ). Huguccio reprend en partie cette explication (ms. Paris, BnF, latin 15396, $\mathrm{f}^{\circ} 188 \mathrm{r}^{\circ} \mathrm{a}$ ).

60. Ainsi dans la rubrique de IIa pars, C 9, q 2, c 1 (F I 602) : « Alterius ecclesiae parrochianum nullus iudicare presumat uel excommunicare ».

61. Décret, IIa pars, C 9, q 2, c 4 : F I 603.

62. Cf. M. Lauwers, «Territorium non facere diocesim... », loc. cit.

63. "Quid sit parochia. Locus in quo degit populus alicui ecclesiae deputatus, certis finibus limitatus, et accipitur hic parochia quatenus spirituale ius ecclesiae se extendit, et in una determinatione plures baptismales esse non possunt, XVI q 1. Plures baptismales imo diuidi debent, et limitatio a Dionysio initium habuit. » (Hostiensis, Summa aurea, lib. III (De parochiis), Lyon, 1537, fo $169 \mathrm{v}^{\circ}$.)

64. Selon Honorius III : « per libros antiquos uel alio modo melius probabuntur, necnon et testes, famam et quaecumque alia adminicula ", repris dans le recueil des décrétales de Grégoire IX (Extra 2, 19, 13). La Glose ordinaire au recueil des décrétales de Grégoire IX explique que la recherche des limites doit être menée de la même manière pour les diocèses et les paroisses.

65. Sur ces questions, voir P. Marchetti, De iure finium. Diritto e confini tra tardo Medievo ed età moderna, Milan, 2001, passim.

66. "Quis intelligatur parochianus. Dicitur autem quis parochianus ratione domicilii. Unde si quis transfert domicilium, parochianus erit illius ecclesie ad quam transfertur ». Le cas d'un fidèle résidant en divers lieux est évoqué : « Si in diuersis locis equaliter degit utriusque ecclesie parochianus intelligitur. » Ainsi donc que celui du voyageur : " Viator uero cuiuslibet ecclesie ad quam uenit parochianus intelligitur. » (Hostiensis, Summa aurea, op. cit., $\mathrm{f}^{\circ} 170 \mathrm{r}^{\circ}$.)

67. «In quibus consistit ius parochiale. In cura animarum parochianorum, de quibus parochialis sacerdos coram Deo tenetur reddere rationem [...]. Item consistit in decimis, primitiis [...]. In cimiterio et iure sepulture [...]. In penitentiis audiendis [...]. In prouentibus qualitercumque prouenientibus [...]. In oblationibus. Unde parochiani singulis diebus dominicis et festiuis debent ad propriam ecclesiam conuenire » (Ibid., fol. $\left.169 \mathrm{v}^{\circ}\right)$.

68. M. Maccarrone, «Cura animarum e parochialis sacerdos nelle costituzioni del IV concilio lateranense (1215). Applicazioni in Italia nel sec. xiii », dans Id., Nuovi studi su 
Innocenzo III, Rome, 1995, p. 271-367. Cf. aussi A. MURRAY, « Confession before 1215 », Transactions of the Royal Historical Society, 6th s., 3, Londres, 1993, p. 51-80.

\section{RÉSUMÉS}

L'étude lexicale proposée explore la dimension spatiale des usages du mot parochia. Dans un premier temps, parochia paraît avoir surtout désigné le populus, c'est-à-dire les fidèles d'une église, dont l'assemblée faisait et définissait la plebs. C'est alors l'autorité épiscopale exercée sur un certain nombre de plebes qui donnait consistance au diocèse. À partir du Ix ${ }^{\mathrm{e}}$ siècle, l'idée s'imposa en outre qu'à tout lieu de culte devait correspondre une aire de prélèvement de la dîme (parfois qualifiée de parochia, souvent désignée par le mot terminum). Les fidèles dépendant d'une église - et s'inscrivant désormais au sein d'un espace plus ou moins circonscrit - furent à cette époque qualifiés de "paroissiens" (parochiani). Les attestations de parochia entre le $\mathrm{IX}^{\mathrm{e}}$ et le $\mathrm{XII}^{\mathrm{e}}$ siècle font apparaître que la "paroisse" fut alors progressivement comprise comme une structure spatiale assurant aux populations des lieux de culte de proximité.

Parish, parishioners and territories. Remarks on the word "parochia» in medieval Latin sources. This lexical study analyses the spatial implications of the word «parochia ». It was originally used to designate the populus, i.e. the faithful belonging to a church, the congregation of which constituted the plebs. The diocese was defined then only by the domination exercised by a bishop over a number of plebes. From the 9th century onwards, the idea that each church was entitled to a tithing area - sometimes designated by the word «parochia», but often by «terminum »became widespread. The faithful belonging to a church, and dwelling within a more or less bounded space, were then called "parishioners" (parochiani). Evidence for the use of the word parochia, between the 9th and the 12th centuries, shows that it was then progressively understood as a spatial entity, which provided communities with churches within a convenient distance.

\section{INDEX}

Keywords : territory, parishioners

Mots-clés : Parochia, paroissien, territoire

\section{AUTEUR}

\section{MICHEL LAUWERS}

Université Nice-Sophia-Antipolis, UFR Lettres, arts et sciences humaines, Département d'histoire, 98, boulevard Édouard Herriot, BP 3209, F-06204 Nice cedex 3 